\title{
Necessary and Sufficient Conditions for Schur Geometrical Convexity of the Four-Parameter Homogeneous Means
}

\author{
Zhen-Hang Yang \\ System Division, Zhejiang Province Electric Power Test and Research Institute, \\ Hangzhou, Zhejiang 310014, China \\ Correspondence should be addressed to Zhen-Hang Yang, yzhkm@163.com \\ Received 20 February 2010; Revised 17 March 2010; Accepted 23 March 2010 \\ Academic Editor: Roman Šimon Hilscher
}

Copyright (C 2010 Zhen-Hang Yang. This is an open access article distributed under the Creative Commons Attribution License, which permits unrestricted use, distribution, and reproduction in any medium, provided the original work is properly cited.

The necessary and sufficient conditions for Schur geometrical convexity of the four-parameter means are given. This gives a unified treatment for Schur geometrical convexity of Stolarsky and Gini means.

\section{Introduction and Main Result}

Let $p, q \in \mathbb{R}$ and $a, b>0$. For $a \neq b$ the Stolarsky means are defined as

$$
S_{p, q}(a, b)= \begin{cases}\left(\frac{q}{p} \frac{a^{p}-b^{p}}{a^{q}-b^{q}}\right)^{1 /(p-q)}, & p q(p-q) \neq 0, \\ L^{1 / p}\left(a^{p}, b^{p}\right), & p \neq 0, q=0, \\ L^{1 / q}\left(a^{q}, b^{q}\right), & q \neq 0, p=0, \\ I^{1 / p}\left(a^{p}, b^{p}\right), & p=q \neq 0, \\ \sqrt{a b}, & p=q=0,\end{cases}
$$


and $S_{p, q}(a, a)=a($ see $[1])$, where

$$
\begin{aligned}
& L(x, y)= \begin{cases}\frac{x-y}{\ln x-\ln y}, & x \neq y, \\
x & x=y,\end{cases} \\
& I(x, y)= \begin{cases}\left(\frac{x^{x}}{y^{y}}\right)^{1 /(x-y)}, & x \neq y, \\
x, & x=y\end{cases}
\end{aligned}
$$

are the logarithmic mean and identric (exponential) mean of positive numbers $x$ and $y$, respectively.

Another two-parameter family of means was introduced by Gini in [2]. That are defined as

$$
G_{p, q}(a, b)= \begin{cases}\left(\frac{a^{p}+b^{p}}{a^{q}+b^{q}}\right)^{1 /(p-q)}, & p \neq q, \\ \exp \left(\frac{a^{p} \ln a+b^{p} \ln b}{a^{p}+b^{p}}\right), & p=q .\end{cases}
$$

Stolarsky and Gini means both are contained in the so-called four-parameter means [3], which are defined as follows.

Definition 1.1. Let $(a, b) \in \mathbb{R}_{+} \times \mathbb{R}_{+}$with $a \neq b$ and $(p, q),(r, s) \in \mathbb{R} \times \mathbb{R}$. Then the four-parameter homogeneous means denoted by $\mathbf{F}(p, q ; r, s ; a, b)$ are defined as follows:

$$
\mathbf{F}(p, q ; r, s ; a, b)=\left(\frac{L\left(a^{p r}, b^{p r}\right)}{L\left(a^{p s}, b^{p s}\right)} \frac{L\left(a^{q s}, b^{q s}\right)}{L\left(a^{q r}, b^{q r}\right)}\right)^{1 /(p-q)(r-s)} \quad \text { if } \operatorname{pqrs}(p-q)(r-s) \neq 0,
$$

or

$$
\mathbf{F}(p, q ; r, s ; a, b)=\left(\frac{a^{p r}-b^{p r}}{a^{p s}-b^{p s}} \frac{a^{q s}-b^{q s}}{a^{q r}-b^{q r}}\right)^{1 /(p-q)(r-s)} \quad \text { if } \operatorname{pqrs}(p-q)(r-s) \neq 0 .
$$

If $\operatorname{pqrs}(p-q)(r-s)=0$, then $\mathbf{F}(p, q ; r, s ; a, b)$ are defined as their corresponding limits, for example:

$$
\begin{gathered}
\mathbf{F}(p, p ; r, s ; a, b)=\lim _{q \rightarrow p} \mathbf{F}(p, q ; r, s ; a, b)=\left(\frac{I\left(a^{p r}, b^{p r}\right)}{I\left(a^{p s}, b^{p s}\right)}\right)^{1 / p(r-s)}, \quad \text { if } p r s(r-s) \neq 0, p=q, \\
\mathbf{F}(p, 0 ; r, s ; a, b)=\lim _{q \rightarrow 0} \mathbf{F}(p, q ; r, s ; a, b)=\left(\frac{L\left(a^{p r}, b^{p r}\right)}{L\left(a^{p s}, b^{p s}\right)}\right)^{1 / p(r-s)}, \quad \text { if } \operatorname{prs}(r-s) \neq 0, q=0, \\
\mathbf{F}(0,0 ; r, s ; a, b)=\lim _{p \rightarrow 0} \mathbf{F}(p, 0 ; r, s ; a, b)=G(a, b), \quad \text { if } r s(r-s) \neq 0, p=q=0,
\end{gathered}
$$


where $L(x, y), I(x, y)$ denote logarithmic mean and identric (exponential) mean, respectively, $G(a, b)=\sqrt{a b}$.

The Schur convexity of $S_{p, q}(a, b)$ and $G_{p, q}(a, b)$ on $(0, \infty) \times(0, \infty)$ with respect to $(a, b)$ was investigated by Qi et al. [4], Shi et al. [5], Li and Shi [6], and Chu and Zhang [7]. Until now, they have been perfectly solved by Chu and Zhang [7], Wang and Zhang [8], respectively. Recently, Chu and Xia also proved the same result as Wang and Zhang [9].

The Schur convexity of $S_{p, q}(a, b)$ and $G_{p, q}(a, b)$ on $[0, \infty) \times[0, \infty)$ and $(-\infty, 0] \times(-\infty, 0]$ with respect to $(p, q)$ was investigated by Qi [10] and Sándor [11], respectively. Now Schur convexity of a four-parameter homogeneous means family containing Stolarsky and Gini means on $(-\infty, \infty) \times(-\infty, \infty)$ with respect to $(p, q)$ has been perfectly solved by Yang [12].

The Schur geometrical convexity was introduced by Zhang [13]. In [8, 14], Wand and Zhang proved that $G_{p, q}(a, b)$ is Schur geometrically convex (Schur geometrically concave) on $(0, \infty) \times(0, \infty)$ with respect to $(a, b)$ if $p+q \geq(\leq) 0$. Chu et al. [15] pointed out that this conclusion is also true for $S_{p, q}(a, b)$. Shi et al. [5, 16], Li and Shi [6], and Gu and Shi [17] also obtained similar results.

The purpose of this paper is to present the necessary and sufficient conditions for Schur geometrical convexity of the four-parameter homogeneous means. This gives a unified treatment for Schur geometrical convexity of Stolarsky and Gini means with respect to $(a, b)$.

Our main result is as follows.

Theorem 1.2. For fixed $(p, q),(r, s) \in \mathbb{R} \times \mathbb{R}$ the four-parameter homogeneous means $\mathbf{F}(p, q ; r, s ; a, b)$ are Schur geometrically convex (Schur geometrically concave) on $(0, \infty) \times(0, \infty)$ with respect to $(a, b)$ if and only if $(p+q)(r+s)>(<) 0$.

\section{Definitions and Lemmas}

Definition 2.1 (see $[18,19])$. Let $\mathbf{x}=\left(x_{1}, x_{2}, \ldots, x_{n}\right)$ and $\mathbf{y}=\left(y_{1}, y_{2}, \ldots, y_{n}\right) \in \mathbb{R}^{n}(n \geq 2)$.

(i) $x$ is said to by majorized by $y$ (in symbol $\mathbf{x}<\mathbf{y}$ ) if

$$
\sum_{i=1}^{k} x_{[i]} \leq \sum_{i=1}^{k} y_{[i]} \quad \text { for } 1 \leq k \leq n-1, \quad \sum_{i=1}^{n} x_{[i]}=\sum_{i=1}^{n} y_{[i]}
$$

where $x_{[1]} \geq x_{[2]} \cdots \geq x_{[n]}$ and $y_{[1]} \geq y_{[2]} \cdots \geq y_{[n]}$ are rearrangements of $\mathbf{x}$ and $\mathbf{y}$ in a decreasing order.

(ii) $\mathbf{x} \geq \mathbf{y}$ means $x_{i} \geq y_{i}$ for all $i=1,2, \ldots, n$. Let $\Omega \subset \mathbb{R}^{n}(n \geq 2)$. The function $\phi: \Omega \rightarrow \mathbb{R}$ is said to be increasing if $\mathbf{x} \geq \mathbf{y}$ implies $\phi(\mathbf{x}) \geq \phi(\mathbf{y})$. $\phi$ is said to be decreasing if and only if $-\phi$ is increasing.

(iii) $\Omega \subset \mathbb{R}^{n}$ is called a convex set if $\left(\alpha x_{1}+\beta y_{1}, \ldots, \alpha x_{n}+\beta y_{n}\right) \in \Omega$ for all $\mathbf{x}$ and $\mathbf{y}$, where $\alpha, \beta \in[0,1]$ with $\alpha+\beta=1$.

(iv) Let $\Omega \subset \mathbb{R}^{n}(n \geq 2)$ be a set with nonempty interior. Then $\phi: \Omega \rightarrow \mathbb{R}$ is said to be Schur convex if $\mathbf{x}<\mathbf{y}$ on $\Omega$ implies $\phi(\mathbf{x}) \leq \phi(\mathbf{y})$. $\phi$ is said to be Schur concave if $-\phi$ is Schur convex. 
Definition 2.2 (see $[13,20])$. Let $\mathbf{x}=\left(x_{1}, x_{2}, \ldots, x_{n}\right)$ and $\mathbf{y}=\left(y_{1}, y_{2}, \ldots, y_{n}\right) \in \mathbb{R}_{+}^{n}(n \geq 2)$. Denote

$$
\ln \mathbf{x}=\left(\ln x_{1}, \ln x_{2}, \ldots, \ln x_{n}\right), \quad \ln \mathbf{y}=\left(\ln y_{1}, \ln y_{2}, \ldots, \ln y_{n}\right)
$$

(i) $\Omega \subset \mathbb{R}_{+}^{n}$ is called a geometrically convex set if $\left(x_{1}^{\alpha} y_{1}^{\beta}, \ldots, x_{n}^{\alpha} y_{n}^{\beta}\right) \in \Omega$ for all $\mathbf{x}$ and $\mathbf{y}$, where $\alpha, \beta \in[0,1]$ with $\alpha+\beta=1$.

(ii) Let $\Omega \subset \mathbb{R}_{+}^{n}(n \geq 2)$ be a set with nonempty interior. Then function $\phi: \Omega \rightarrow \mathbb{R}_{+}$is said to be Schur geometrically convex on $\Omega$ if $\ln \mathbf{x} \prec \ln \mathbf{y}$ on $\Omega$ implies $\phi(\mathbf{x}) \leq \phi(\mathbf{y})$. $\phi$ is said to be Schur geometrically concave if $-\phi$ is Schur geometrically convex.

Definition 2.3 (see [18]). (i) $\Omega \subset \mathbb{R}^{n}(n \geq 2)$ is called symmetric set if $\mathbf{x} \in \Omega$ implies $P \mathbf{x} \in \Omega$ for every $n \times n$ permutation matrix $P$.

(ii) The function $\phi: \Omega \rightarrow \mathbb{R}$ is called symmetric if for every permutation matrix $P$, $\phi(P \mathbf{x})=\phi(\mathbf{x})$ for all $\mathbf{x} \in \Omega$.

Lemma 2.4 (see $[18,19]$ ). Let $\Omega \subset \mathbb{R}^{n}$ be a symmetric set with nonempty interior $\Omega^{0}$ and $\phi: \Omega \rightarrow$ $\mathbb{R}$ be continuous on $\Omega$ and differentiable in $\Omega^{0}$. Then $\phi$ is Schur convex (Schur concave) on $\Omega$ if and only if $\phi$ is symmetric on $\Omega$ and

$$
\left(x_{1}-x_{2}\right)\left(\frac{\partial \phi}{\partial x_{1}}-\frac{\partial \phi}{\partial x_{2}}\right) \geq(\leq) 0
$$

holds for any $\mathbf{x}=\left(x_{1}, x_{2}, \ldots, x_{n}\right) \in \Omega^{0}$.

Lemma 2.5 (see [13, Theorem 1.4, page 108]). Let $\Omega \subset \mathbb{R}_{+}^{n}$ be a symmetric set with a nonempty interior geometrically convex set $\Omega^{0}$. Let $\phi: \Omega \rightarrow \mathbb{R}_{+}$be continuous on $\Omega$ and differentiable in $\Omega^{0}$. Then $\phi$ is Schur geometrically convex (Schur geometrically concave) on $\Omega$ if and only if $\phi$ is symmetric on $\Omega$ and

$$
\left(\ln x_{1}-\ln x_{2}\right)\left(x_{1} \frac{\partial \phi}{\partial x_{1}}-x_{2} \frac{\partial \phi}{\partial x_{2}}\right) \geq(\leq) 0
$$

holds for any $\mathbf{x}=\left(x_{1}, x_{2}, \ldots, x_{n}\right) \in \Omega^{0}$.

\section{Schur Geometrical Convexity of Two-Parameter Homogeneous Functions}

The more general form of two-parameter homogeneous means is the so-called two-parameter homogenous functions first introduced by Yang [21]. For conveniences, we record it as follows.

Definition 3.1. Assume that $f: \mathbb{R}_{+} \times \mathbb{R}_{+} \rightarrow \mathbb{R}_{+} \cup\{0\}$ is $n$-order homogeneous, continuous and exists first partial derivatives and $(a, b) \in \mathbb{R}_{+} \times \mathbb{R}_{+},(p, q) \in \mathbb{R} \times \mathbb{R}$. 
If $f(x, y)>0$ for $(x, y) \in \mathbb{R}_{+} \times \mathbb{R}_{+} \backslash\left\{(x, x): x \in \mathbb{R}_{+}\right\}$and $f(x, x)=0$ for all $x \in \mathbb{R}_{+}$, then define

$$
\begin{gathered}
\mathscr{\ell}_{f}(p, q ; a, b)=\left(\frac{f\left(a^{p}, b^{p}\right)}{f\left(a^{q}, b^{q}\right)}\right)^{1(p-q)} \text { if } p \neq q, p q \neq 0, \\
\mathscr{H}_{f}(p, p ; a, b)=\lim _{q \rightarrow p} \mathscr{L}_{f}(p, q ; a, b)=G_{f, p}(a, b) \text { if } p=q \neq 0,
\end{gathered}
$$

where

$$
G_{f, p}(a, b)=G_{f}^{1 / p}\left(a^{p}, b^{p}\right), \quad G_{f}(x, y)=\exp \left(\frac{x f_{x}(x, y) \ln x+y f_{y}(x, y) \ln y}{f(x, y)}\right),
$$

$f_{x}(x, y)$ and $f_{y}(x, y)$ denote first-order partial derivatives with respect to first and second component of $f(x, y)$, respectively.

If $f(x, y)>0$ for all $(x, y) \in \mathbb{R}_{+} \times \mathbb{R}_{+}$, then define further

$$
\begin{gathered}
\mathscr{H}_{f}(p, 0 ; a, b)=\left(\frac{f\left(a^{p}, b^{p}\right)}{f(1,1)}\right)^{1 / p} \text { if } p \neq 0, q=0 ; \\
\mathscr{H}_{f}(0, q ; a, b)=\left(\frac{f\left(a^{q}, b^{q}\right)}{f(1,1)}\right)^{1 / q} \text { if } p=0, q \neq 0 ; \\
\mathscr{H}_{f}(0,0 ; a, b)=a^{f_{x}(1,1) / f(1,1)} b^{f_{y}(1,1) / f(1,1)} \text { if } p=q=0 .
\end{gathered}
$$

Since $f(x, y)$ is a homogeneous function, $\mathscr{\ell}_{f}(p, q ; a, b)$ is also one and called a homogeneous function with parameters $p$ and $q$ and simply denoted by $\mathscr{L}_{f}(p, q)$ or $\mathfrak{l}_{f}$ sometimes.

Concerning the monotonicity and log-convexity of two-parameter homogeneous functions, there have been some literatures such as [3,21,22], which yield some new and interesting inequalities for means.

The two-parameter homogeneous functions $\mathscr{L}_{f}(p, q ; a, b)$ have some well properties (see [21-23]) such as the following lemma.

Lemma 3.2 (see [23]). Let $f: \mathbb{R}_{+} \times \mathbb{R}_{+} \rightarrow \mathbb{R}_{+}$be a homogenous and differentiable function and

$$
T(t)=T(t ; a, b):=\ln f\left(a^{t}, b^{t}\right), \quad(t ; a, b) \in \mathbb{R} \times \mathbb{R}_{+} \times \mathbb{R}_{+} .
$$

Then we have

$$
\begin{aligned}
& \frac{\partial T(t ; a, b)}{\partial t}=\frac{a^{t} f_{x}\left(a^{t}, b^{t}\right) \ln a+b^{t} f_{y}\left(a^{t}, b^{t}\right) \ln b}{f\left(a^{t}, b^{t}\right)} \\
& \ln \mathscr{\ell}_{f}(p, q ; a, b)=\int_{0}^{1} \frac{\partial T(t p+(1-t) q ; a, b)}{\partial t} d t .
\end{aligned}
$$

Next we give another property. 
Lemma 3.3. Let $f: \mathbb{R}_{+} \times \mathbb{R}_{+} \rightarrow \mathbb{R}_{+}$be a homogenous and m-time differentiable function. Then $\mathscr{H}_{f}(p, q ; a, b) \in C^{m-1}\left(\mathbb{R} \times \mathbb{R} \times \mathbb{R}_{+} \times \mathbb{R}_{+}\right)$.

Proof. Since $f(x, y)$ has continuous partial derivatives of $m$ order with respect to $x, y$ on $\mathbb{R}_{+} \times$ $\mathbb{R}_{+}$, the integrand in (3.6) has continuous partial derivatives of $m-1$ order with respect to $p, q, a, b$ on $\mathbb{R} \times \mathbb{R} \times \mathbb{R}_{+} \times \mathbb{R}_{+}$, that is $\mathscr{\ell}_{f}(p, q ; a, b) \in C^{m-1}\left(\mathbb{R} \times \mathbb{R} \times \mathbb{R}_{+} \times \mathbb{R}_{+}\right)$.

For the Schur geometrical convexity, we have the following result.

Theorem 3.4. Assume that $f: \mathbb{R}_{+} \times \mathbb{R}_{+} \rightarrow \mathbb{R}_{+}$is a symmetric, n-order homogeneous, continuous, and three-time differentiable function. If for any $(x, y) \in \mathbb{R}_{+} \times \mathbb{R}_{+}$with $x \neq y$

$$
\mathcal{N}(x, y)=(x-y)\left(x(\ln f)_{x}-y(\ln f)_{y}-2 x y \supset \ln \left(\frac{x}{y}\right)\right)>(<) 0, \quad \text { where } \supset=(\ln f)_{x y^{\prime}}
$$

then $\mathscr{L}_{f}(p, q ; a, b)$ is Schur geometrically convex on $(0, \infty) \times(0, \infty)$ with respect to $(a, b)$ if and only if $p+q>(<) 0$ and Schur geometrically concave if and only if $p+q<(>) 0$.

Proof. (1) In the case of $p \neq q$. We have

$$
\ln \mathscr{d}_{f}(p, q ; a, b)=\frac{\ln f\left(a^{p}, b^{p}\right)-\ln f\left(a^{q}, b^{q}\right)}{p-q} .
$$

Some simple partial derivative computations yield

$$
\begin{aligned}
& \frac{\partial \ln \mathscr{H}_{f}}{\partial a}=\frac{1}{\mathscr{l}_{f}} \frac{\partial \mathscr{H}_{f}}{\partial a}=\frac{1}{p-q}\left(\frac{p a^{p-1} f_{x}\left(a^{p}, b^{p}\right)}{f\left(a^{p}, b^{p}\right)}-\frac{q a^{q-1} f_{x}\left(a^{q}, b^{q}\right)}{f\left(a^{q}, b^{q}\right)}\right), \\
& \frac{\partial \ln \mathscr{L}_{f}}{\partial b}=\frac{1}{\mathscr{l}_{f}} \frac{\partial \mathscr{\ell}_{f}}{\partial b}=\frac{1}{p-q}\left(\frac{p b^{p-1} f_{y}\left(a^{p}, b^{p}\right)}{f\left(a^{p}, b^{p}\right)}-\frac{q b^{q-1} f_{y}\left(a^{q}, b^{q}\right)}{f\left(a^{q}, b^{q}\right)}\right),
\end{aligned}
$$

hence,

$$
\frac{1}{\mathscr{\ell}_{f}}\left(a \frac{\partial \mathscr{\ell}_{f}}{\partial a}-b \frac{\partial \mathscr{\ell}_{f}}{\partial b}\right)=\frac{g(p)-g(q)}{p-q}
$$

where

$$
g(t)=\frac{t a^{t} f_{x}\left(a^{t}, b^{t}\right)}{f\left(a^{t}, b^{t}\right)}-\frac{t b^{t} f_{y}\left(a^{t}, b^{t}\right)}{f\left(a^{t}, b^{t}\right)}
$$

It is easy to verify that $g(t)$ is even on $(-\infty, \infty)$. In fact, since $f(x, y)$ is $n$-order homogeneous and symmetric, for arbitrary $\lambda>0$, we have

$$
\begin{gathered}
f(\lambda x, \lambda y)=\lambda^{n} f(x, y), \quad f_{x}(\lambda x, \lambda y)=\lambda^{n-1} f_{x}(x, y), \quad f_{y}(\lambda x, \lambda y)=\lambda^{n-1} f_{y}(x, y), \\
f(x, y)=f(y, x), \quad f_{x}(x, y)=f_{y}(y, x), \quad f_{y}(x, y)=f_{x}(y, x) .
\end{gathered}
$$


Thus,

$$
\begin{aligned}
g(-t) & =\frac{-t a^{-t} f_{x}\left(a^{-t}, b^{-t}\right)}{f\left(a^{-t}, b^{-t}\right)}-\frac{-t b^{-t} f_{y}\left(a^{-t}, b^{-t}\right)}{f\left(a^{-t}, b^{-t}\right)} \\
& =\frac{-t a^{-t}\left(a^{t} b^{t}\right)^{-(n-1)} f_{x}\left(b^{t}, a^{t}\right)}{\left(a^{t} b^{t}\right)^{-n} f\left(b^{t}, a^{t}\right)}-\frac{-t b^{t}\left(a^{t} b^{t}\right)^{-(n-1)} f_{y}\left(b^{t}, a^{t}\right)}{\left(a^{t} b^{t}\right)^{-n} f\left(b^{t}, a^{t}\right)} \\
& =-\frac{t b^{t} f_{y}\left(a^{t}, b^{t}\right)}{f\left(a^{t}, b^{t}\right)}+\frac{t a^{t} f_{x}\left(a^{t}, b^{t}\right)}{f\left(a^{t}, b^{t}\right)}=g(t) .
\end{aligned}
$$

Let $a^{t}=x, b^{t}=y$. Then

$$
\begin{aligned}
g^{\prime}(t)= & x(\ln f)_{x}+t\left(\left(\frac{x f_{x}(x, y)}{f(x, y)}\right)_{x} \frac{d x}{d t}+\left(\frac{x f_{x}(x, y)}{f(x, y)}\right)_{y} \frac{d y}{d t}\right) \\
& -y(\ln f)_{y}-t\left(\left(\frac{y f_{y}(x, y)}{f(x, y)}\right)_{x} \frac{d x}{d t}+\left(\frac{y f_{y}(x, y)}{f(x, y)}\right)_{y} \frac{d y}{d t}\right) \\
= & x(\ln f)_{x}+t\left(x\left(\frac{x f_{x}(x, y)}{f(x, y)}\right)_{x} \ln a+y\left(\frac{x f_{x}(x, y)}{f(x, y)}\right)_{y} \ln b\right) \\
& -y(\ln f)_{y}-t\left(x\left(\frac{y f_{y}(x, y)}{f(x, y)}\right)_{x} \ln a+y\left(\frac{y f_{y}(x, y)}{f(x, y)}\right)_{y} \ln b\right) .
\end{aligned}
$$

Note $x f_{x}(x, y) / f(x, y)$ and $y f_{y}(x, y) / f(x, y)$ both are 0 -order homogeneous with respect to $x$ and $y$, then

$$
\begin{gathered}
x\left(\frac{x f_{x}(x, y)}{f(x, y)}\right)_{x}+y\left(\frac{x f_{x}(x, y)}{f(x, y)}\right)_{y}=0 \\
x\left(\frac{y f_{y}(x, y)}{f(x, y)}\right)_{x}+y\left(\frac{y f_{y}(x, y)}{f(x, y)}\right)_{y}=0
\end{gathered}
$$

and then

$$
\begin{gathered}
x\left(\frac{x f_{x}(x, y)}{f(x, y)}\right)_{x}=-y\left(\frac{x f_{x}(x, y)}{f(x, y)}\right)_{y}=-x y \supset, \\
y\left(\frac{y f_{y}(x, y)}{f(x, y)}\right)_{y}=-x\left(\frac{y f_{y}(x, y)}{f(x, y)}\right)_{x}=-x y \supset .
\end{gathered}
$$


Therefore,

$$
\begin{aligned}
g^{\prime}(t) & =x(\ln f)_{x}+t x y \supset(\ln b-\ln a)-y(\ln f)_{y}-t x y \supset(\ln a-\ln b) \\
& =x(\ln f)_{x}-y(\ln f)_{y}-2 t x y \supset(\ln a-\ln b) \\
& =x(\ln f)_{x}-y(\ln f)_{y}-2 x y \supset \ln \left(\frac{x}{y}\right)=\frac{N(x, y)}{x-y} \quad \text { for } x \neq y
\end{aligned}
$$

By the mean values theorem, there is a $\xi$ between $|p|$ and $|q|$ such that

$$
\frac{g(p)-g(q)}{p-q}=\frac{g(|p|)-g(|q|)}{p-q}=\frac{|p|-|q|}{p-q} g^{\prime}(\xi)=\frac{p+q}{|p|+|q|} g^{\prime}(\xi)=\frac{p+q}{|p|+|q|} \frac{N(x, y)}{x-y}, \quad \text { for } x \neq y
$$

where $x=a^{\xi}, y=b^{\xi}$. Thus we have

$$
\begin{aligned}
(\ln a-\ln b)\left(a \frac{\partial \mathscr{L}_{f}}{\partial a}-b \frac{\partial \mathscr{L}_{f}}{\partial b}\right) & =\mathscr{L}_{f} \frac{p+q}{|p|+|q|} \ln \left(\frac{a}{b}\right) \frac{\mathcal{N}(x, y)}{x-y} \\
& =\mathscr{H}_{f} \frac{p+q}{|p|+|q|} \frac{\mathcal{N}(x, y)}{\xi} \frac{\ln x-\ln y}{x-y} \\
& = \begin{cases}>0 & \text { if } p+q>(<) 0 \\
<0 & \text { if } p+q<(>) 0 .\end{cases}
\end{aligned}
$$

By Lemma 2.5, our required result is derived immediately.

(2) In the case of $p=q \neq 0$. By Lemma 3.3 together with (3.10) and (3.17), we have

$$
\begin{aligned}
\frac{1}{\mathscr{L}_{f}(p, p)}\left(a \frac{\partial \mathscr{L}_{f}(p, p)}{\partial a}-b \frac{\partial \mathscr{\ell}_{f}(p, p)}{\partial b}\right) & =\lim _{q \rightarrow p} \frac{1}{\mathscr{L}_{f}(p, q)}\left(a \frac{\partial \mathscr{L}_{f}(p, q)}{\partial a}-b \frac{\partial \mathscr{L}_{f}(p, q)}{\partial b}\right) \\
& =\lim _{q \rightarrow p} \frac{g(p)-g(q)}{p-q}=g^{\prime}(p)=\frac{N(x, y)}{x-y},
\end{aligned}
$$


where $x=a^{p}, y=b^{p}$. Hence we have

$$
\begin{aligned}
(\ln a-\ln b)\left(a \frac{\partial \mathscr{L}_{f}(p, p)}{\partial a}-b \frac{\partial \mathscr{L}_{f}(p, p)}{\partial b}\right) & =\mathscr{H}_{f}(p, p)(\ln a-\ln b) \frac{\mathcal{N}(x, y)}{x-y} \\
& =p^{-1} \mathscr{H}_{f}(p, p) \mathcal{N}(x, y) \frac{\ln x-\ln y}{x-y} \\
& = \begin{cases}>0 & \text { if } p>(<) 0, \\
<0 & \text { if } p<(>) 0 .\end{cases}
\end{aligned}
$$

By Lemma 2.5, the required result holds.

(3) In the case of $p=q=0$. By Lemma 3.3 and (3.20), we have

$$
\frac{1}{\mathscr{H}_{f}(0,0)}\left(a \frac{\partial \mathscr{H}_{f}(0,0)}{\partial a}-b \frac{\partial \mathscr{H}_{f}(0,0)}{\partial b}\right)=\lim _{p \rightarrow 0}\left(a \frac{\partial \mathscr{L}_{f}(p, p)}{\partial a}-b \frac{\partial \mathscr{H}_{f}(p, p)}{\partial b}\right)=\lim _{p \rightarrow 0} g^{\prime}(p)
$$

However,

$$
\begin{aligned}
g^{\prime}(0) & =\left.\left(x(\ln f)_{x}-y(\ln f)_{y}-2 x y \supset \ln \left(\frac{x}{y}\right)\right)\right|_{x=1, y=1} \\
& =1 \cdot \frac{f_{x}(1,1)}{f(1,1)}-1 \cdot \frac{f_{y}(1,1)}{f(1,1)}-2 \cdot 1 \cdot 1 \cdot \supset(1,1) \cdot \ln \left(\frac{1}{1}\right)=0,
\end{aligned}
$$

where $f_{x}(1,1)=f_{y}(1,1)$ due to the symmetry of $f(x, y)$. Thus

$$
(\ln a-\ln b)\left(a \frac{\partial \mathscr{L}_{f}(p, p)}{\partial a}-b \frac{\partial \mathscr{L}_{f}(p, p)}{\partial b}\right)=0
$$

Summarizing the above three cases, this proof of Theorem 3.4 is complete. 


\section{Proof of Main Result}

Establishing the Theorem 3.4, we are in a position to prove main result.

Proof of Theorem 1.2. It follows from [3, Section 1], that $\mathbf{F}(p, q ; r, s ; a, b)=\mathscr{\ell}_{\mathfrak{d}_{L}}(p, q ; a, b)$, where $\mathscr{d}_{L}=\mathscr{\ell}_{L}(r, s)=\mathscr{L}_{L}(r, s ; x, y)=S_{r, s}(x, y)$ is symmetric with respect to $x$ and $y$. From Lemma 3.3, it follows that $\mathscr{L}_{L}=\mathscr{L}_{L}(r, s ; x, y) \in C^{\infty}\left(\mathbb{R} \times \mathbb{R} \times \mathbb{R}_{+} \times \mathbb{R}_{+}\right)$. Thus we have

$$
\begin{aligned}
& \left(\ln \mathscr{L}_{L}(r, r)\right)_{x}=\lim _{s \rightarrow r}\left(\ln \mathscr{\ell}_{L}(r, s)\right)_{x} \\
& \left(\ln \mathscr{L}_{L}(r, r)\right)_{y}=\lim _{s \rightarrow r}\left(\ln \mathscr{L}_{L}(r, s)\right)_{y^{\prime}} \\
& \left(\ln \mathscr{L}_{L}(r, r)\right)_{x y}=\lim _{s \rightarrow r}\left(\ln \mathscr{L}_{L}(r, s)\right)_{x y}, \\
& \left(\ln \mathscr{d}_{L}(r, 0)\right)_{x}=\lim _{s \rightarrow 0}\left(\ln \mathscr{d}_{L}(r, s)\right)_{x} \\
& \left(\ln \mathscr{L}_{L}(r, 0)\right)_{y}=\lim _{s \rightarrow 0}\left(\ln \mathscr{L}_{L}(r, s)\right)_{y^{\prime}} \\
& \left(\ln \mathscr{L}_{L}(r, 0)\right)_{x y}=\lim _{s \rightarrow r}\left(\ln \mathscr{L}_{L}(r, s)\right)_{x y} \\
& \left(\ln \mathscr{L}_{L}(0,0)\right)_{x}=\lim _{r \rightarrow 0}\left(\ln \mathscr{L}_{L}(r, r)\right)_{x} \\
& \left(\ln \mathscr{d}_{L}(0,0)\right)_{y}=\lim _{r \rightarrow 0}\left(\ln \mathscr{d}_{L}(r, r)\right)_{y^{\prime}} \\
& \left(\ln \mathscr{L}_{L}(0,0)\right)_{x y}=\lim _{r \rightarrow 0}\left(\ln \mathscr{L}_{L}(r, r)\right)_{x y} .
\end{aligned}
$$

(1) In the case of $r s(r-s) \neq 0$.

Simple partial derivative calculations yield

$$
\begin{gathered}
\ln \mathscr{\ell}_{L}=\frac{1}{r-s}\left(\ln |s|+\ln \left|x^{r}-y^{r}\right|-\ln |r|-\ln \left|x^{s}-y^{s}\right|\right) \\
\left(\ln \mathscr{L}_{L}\right)_{x}=\frac{1}{r-s}\left(\frac{r x^{r-1}}{x^{r}-y^{r}}-\frac{s x^{s-1}}{x^{s}-y^{s}}\right) \\
\left(\ln \mathscr{H}_{L}\right)_{y}=\frac{1}{r-s}\left(\frac{-r y^{r-1}}{x^{r}-y^{r}}+\frac{s y^{s-1}}{x^{s}-y^{s}}\right) \\
\supset=\left(\ln \mathscr{H}_{L}\right)_{x y}=\frac{1}{x y(r-s)}\left(\frac{r^{2} x^{r} y^{r}}{\left(x^{r}-y^{r}\right)^{2}}-\frac{s^{2} x^{s} y^{s}}{\left(x^{s}-y^{s}\right)^{2}}\right) .
\end{gathered}
$$


Hence,

$$
\begin{aligned}
\mathcal{N}(x, y)= & (x-y)\left(x\left(\ln \mathscr{d}_{L}\right)_{x}-y\left(\ln \mathscr{\ell}_{L}\right)_{y}-2 x y \supset \ln \left(\frac{x}{y}\right)\right) \\
= & \frac{x-y}{r-s}\left(\frac{r\left(x^{r}+y^{r}\right)}{x^{r}-y^{r}}-\frac{2 r^{2} x^{r} y^{r} \ln (x / y)}{\left(x^{r}-y^{r}\right)^{2}}\right) \\
& -\frac{x-y}{r-s}\left(\frac{s\left(x^{s}+y^{s}\right)}{x^{s}-y^{s}}-\frac{2 s^{2} x^{s} y^{s} \ln (x / y)}{\left(x^{s}-y^{s}\right)^{2}}\right) \\
= & (x-y) \frac{P(r)-P(s)}{r-s},
\end{aligned}
$$

where

$$
P(t)=t\left(\frac{x^{t}+y^{t}}{x^{t}-y^{t}}-\frac{2 x^{t} y^{t} \ln \left(x^{t} / y^{t}\right)}{\left(x^{t}-y^{t}\right)^{2}}\right) .
$$

It is easy to check that $P(t)$ is even and increasing (decreasing) on $(0, \infty)$ if $x>(<) y$. Indeed,

$$
P(-t)=-t\left(\frac{x^{-t}+y^{-t}}{x^{-t}-y^{-t}}-\frac{2 x^{-t} y^{-t} \ln \left(x^{-t} / y^{-t}\right)}{\left(x^{-t}-y^{-t}\right)^{2}}\right)=P(t)
$$

With $(x / y)^{t}=u$, then $t=\ln u / \ln (x / y)$, and then $P(t)$ can be written as

$$
P(t)=\frac{1}{\ln (x / y)}\left(\frac{u+1}{u-1} \ln u-\frac{2 u \ln ^{2} u}{(u-1)^{2}}\right) .
$$

Direct computation yields

$$
\begin{aligned}
P^{\prime}(t) & =\frac{1}{\ln (x / y)}\left(\frac{u+1}{u-1} \ln u-\frac{2 u \ln ^{2} u}{(u-1)^{2}}\right)^{\prime} \frac{d u}{d t} \\
& =u\left((u+1) \frac{(u-1) / u-\ln u}{(u-1)^{2}}+\frac{\ln u}{u-1}-\frac{2 \ln ^{2} u}{(u-1)^{2}}-4 u \frac{\ln u}{u-1} \frac{(u-1) / u-\ln u}{(u-1)^{2}}\right) \\
& =\frac{(u-1) / \ln u=L}{=} \frac{(u+1) L^{2}-6 u L+2 u(u+1)}{(u-1) L^{2}} \\
& =\frac{2 L(((u+1) / 2) L-u)+4 u((u+1) / 2-L)}{(u-1) L^{2}} .
\end{aligned}
$$


From

$$
\begin{gathered}
\frac{u+1}{2} L-u=\frac{u^{2}-1}{\ln u^{2}}-\sqrt{u^{2}}>0, \\
L-\frac{u+1}{2}<0,
\end{gathered}
$$

it follows that $P^{\prime}(t)>0$ if $u-1>0$, that is, $x>y$ and $P^{\prime}(t)<0$ if $x<y$. Namely,

$$
(x-y) P^{\prime}(t)>0 \text { for } t>0 \text { with } x \neq y \text {. }
$$

By the mean values theorem, there is a $\eta$ between $|r|$ and $|s|$ such that

$$
P(|r|)-P(|s|)=(|r|-|s|) P^{\prime}(\eta)
$$

and then

$$
\begin{aligned}
\mathcal{N}(x, y) & =(x-y) \frac{P(r)-P(s)}{r-s}=(x-y) \frac{r+s}{|r|+|s|} \frac{P(|r|)-P(|s|)}{|r|-|s|} \\
& =\frac{r+s}{|r|+|s|} \cdot(x-y) P^{\prime}(\eta) \\
& = \begin{cases}>0 & \text { if } r+s>0, \\
<0 & \text { if } r+s<0 .\end{cases}
\end{aligned}
$$

Using Theorem 3.4, for fixed $(p, q),(r, s) \in \mathbb{R} \times \mathbb{R}$ with $r s(r-s) \neq 0$, the four-parameter homogeneous means $\mathbf{F}(p, q ; r, s ; a, b)$ are Schur geometrically convex on $(0, \infty) \times(0, \infty)$ with respect to $(a, b)$ if and only if $(p+q)(r+s)>0$ and Schur geometrically concave if and only if $(p+q)(r+s)<0$.

(2) In the case of $s=0, r \neq 0$.

From (4.11) together with (4.4)-(4.6) and (4.19), there is a $\eta_{1}$ between 0 and $|r|$ such that

$$
\begin{aligned}
\mathcal{N}(x, y) & =(x-y)\left(x\left(\ln \mathscr{L}_{L}(r, 0)\right)_{x}-y\left(\ln \mathscr{L}_{L}(r, 0)\right)_{y}-2 x y\left(\ln \mathscr{d}_{L}(r, 0)\right)_{x y} \ln \left(\frac{x}{y}\right)\right) \\
& =\lim _{s \rightarrow 0}\left((x-y)\left(x\left(\ln \mathscr{d}_{L}(r, s)\right)_{x}-y\left(\ln \mathscr{d}_{L}(r, s)\right)_{y}-2 x y\left(\ln \mathscr{\ell}_{L}(r, s)\right)_{x y} \ln \left(\frac{x}{y}\right)\right)\right) \\
& =\lim _{s \rightarrow 0}(x-y) \frac{P(r)-P(s)}{r-s}=\lim _{s \rightarrow 0} \frac{r+s}{|r|+|s|} \cdot \lim _{s \rightarrow 0}(x-y) P^{\prime}\left(\eta_{1}\right) \\
& = \begin{cases}>0 & \text { if } r>0, \quad(\text { by }(4.17)) . \\
<0 & \text { if } r<0,\end{cases}
\end{aligned}
$$


(3) In the case of $r=0, s \neq 0$.

Since $\mathfrak{l}_{L}(r, s ; x, y)$ is symmetric with respect to $r$ and $s$, it follows from case 2 that

$$
\begin{aligned}
\mathcal{N}(x, y) & =(x-y)\left(x\left(\ln \mathscr{L}_{L}(0, s)\right)_{x}-y\left(\ln \mathscr{L}_{L}(0, s)\right)_{y}-2 x y\left(\ln \mathscr{L}_{L}(r, s)\right)_{x y} \ln \left(\frac{x}{y}\right)\right) \\
& = \begin{cases}>0 & \text { if } s>0, \\
<0 & \text { if } s<0 .\end{cases}
\end{aligned}
$$

(4) In the case of $r=s \neq 0$.

From (4.11) together with (4.1)-(4.3), we have

$$
\begin{aligned}
\mathcal{N}(x, y) & =(x-y)\left(x\left(\ln \mathscr{L}_{L}(r, r)\right)_{x}-y\left(\ln \mathscr{L}_{L}(r, r)\right)_{y}-2 x y\left(\ln \mathscr{L}_{L}(r, r)\right)_{x y} \ln \left(\frac{x}{y}\right)\right) \\
& =\lim _{s \rightarrow r}\left((x-y)\left(x\left(\ln \mathscr{L}_{L}(r, s)\right)_{x}-y\left(\ln \mathscr{L}_{L}(r, s)\right)_{y}-2 x y\left(\ln \mathscr{L}_{L}(r, s)\right)_{x y} \ln \left(\frac{x}{y}\right)\right)\right) \\
& =(x-y) \lim _{s \rightarrow r} \frac{P(r)-P(s)}{r-s}=(x-y) P^{\prime}(r) \\
& =\left\{\begin{array}{lll}
>0 & \text { if } r>0, \quad(\text { by }(4.17)) \\
<0 & \text { if } r<0 .
\end{array}\right.
\end{aligned}
$$

(5) In the case of $r=s=0$.

From (4.22) together with (4.7)-(4.9), we have

$$
\begin{aligned}
\mathcal{N}(x, y) & =(x-y)\left(x\left(\ln \mathscr{L}_{L}(0,0)\right)_{x}-y\left(\ln \mathscr{L}_{L}(0,0)\right)_{y}-2 x y\left(\ln \mathscr{L}_{L}(0,0)\right)_{x y} \ln \left(\frac{x}{y}\right)\right) \\
& =\lim _{r \rightarrow 0}\left((x-y)\left(x\left(\ln \mathscr{L}_{L}(r, r)\right)_{x}-y\left(\ln \mathscr{L}_{L}(r, r)\right)_{y}-2 x y\left(\ln \mathscr{L}_{L}(r, r)\right)_{x y} \ln \left(\frac{x}{y}\right)\right)\right) \\
& =(x-y) \lim _{r \rightarrow 0} P^{\prime}(r) .
\end{aligned}
$$

But by (4.15) and some limit computations, we obtain

$$
\begin{aligned}
& \lim _{t \rightarrow 0} P^{\prime}(t) \underline{\underline{(x / y)^{t}=u}} \lim _{u \rightarrow 1} u\left((u+1) \frac{(u-1) / u-\ln u}{(u-1)^{2}}+\frac{\ln u}{u-1}-\frac{2 \ln ^{2} u}{(u-1)^{2}}\right. \\
& \left.-4 u \frac{\ln u}{u-1} \frac{(u-1) / u-\ln u}{(u-1)^{2}}\right)=0,
\end{aligned}
$$

which implies $\mathcal{N}(x, y)=0$. 
Summarizing the above five cases, our required results are derived.

This proof ends.

\section{Other Corollaries}

The four-parameter homogeneous means $\mathrm{F}(p, q ; r, s ; a, b)$ also contain many other twoparameter means, for instance, for the identric (exponential) mean defined by (1.3), its twoparameter means are defined as follows [21, Example 2.3]:

$$
\mathscr{H}_{I}(p, q ; a, b)= \begin{cases}\left(\frac{I\left(a^{p}, b^{p}\right)}{I\left(a^{q}, b^{q}\right)}\right)^{1 /(p-q)}, & p \neq q, p q \neq 0, \\ G_{I, p}(a, b), & p=q \neq 0, \\ I^{1 / p}\left(a^{p}, b^{p}\right), & p \neq 0, q=0, \\ I^{1 / q}\left(a^{q}, b^{q}\right), & p=0, q \neq 0, \\ G(a, b), & p=q=0,\end{cases}
$$

where $G_{I, p}(a, b)=Y^{1 / p}\left(a^{p}, b^{p}\right):=Y_{p}(a, b), Y(a, b)=I e^{1-G^{2} / L^{2}}$.

By [3], we see that

$$
\mathscr{L}_{I}(p, q ; a, b)=\mathbf{F}(p, q ; 1,1 ; a, b) .
$$

And then according to Theorem 1.2, we have the following corollary.

Corollary 5.1. For fixed $(p, q) \in \mathbb{R} \times \mathbb{R}$, the two-parameter identric (exponential) means $\mathfrak{d}_{I}(p, q$; $a, b)$ are Schur geometrically convex on $(0, \infty) \times(0, \infty)$ with respect to $(a, b)$ if and only if $p+q>0$ and Schur geometrically concave if and only if $p+q<0$.

As another example, for Heronian mean defined by

$$
\mathrm{He}=\frac{a+\sqrt{a b}+b}{3},
$$

its two-parameter means are defined as follows:

$$
\mathscr{H}_{\mathrm{He}}(p, q ; a, b)= \begin{cases}\left(\frac{a^{p}+(\sqrt{a b})^{p}+b^{p}}{a^{q}+(\sqrt{a b})^{q}+b^{q}}\right)^{1 /(p-q)}, & p \neq q, p q \neq 0, \\ a^{\left(a^{p}+(\sqrt{a b})^{p} / 2\right) /\left(a^{p}+(\sqrt{a b})^{p}+b^{p}\right)} b^{\left((\sqrt{a b})^{p} / 2+b^{p}\right) /\left(a^{p}+(\sqrt{a b})^{p}+b^{p}\right)}, & p=q \neq 0, \\ \mathrm{He}^{1 / p}\left(a^{p}, b^{p}\right), & p \neq 0, q=0, \\ \mathrm{He}^{1 / q}\left(a^{q}, b^{q}\right), & p=0, q \neq 0, \\ G(a, b), & p=q=0 .\end{cases}
$$


By [3], we see that

$$
\mathscr{H}_{\mathrm{He}}(p, q ; \mathrm{a}, b)=\mathbf{F}(p, q ; 3 / 2,1 / 2 ; a, b) .
$$

And then according to Theorem 1.2, we have the following corollary.

Corollary 5.2. For fixed $(p, q) \in \mathbb{R} \times \mathbb{R}$, the two-parameter Heronian means $\mathfrak{d}_{\mathrm{He}}(p, q ; a, b)$ are Schur geometrically convex on $(0, \infty) \times(0, \infty)$ with respect to $(a, b)$ if and only if $p+q>0$ and Schur geometrically concave if and only if $p+q<0$.

\section{References}

[1] K. B. Stolarsky, "Generalizations of the logarithmic mean," Mathematics Magazine, vol. 48, pp. 87-92, 1975.

[2] C. Gini, “Diuna formula comprensiva delle media," Metron, vol. 13, pp. 3-22, 1938.

[3] Zh.-H. Yang, "On the monotonicity and log-convexity of a four-parameter homogeneous mean," Journal of Inequalities and Applications, vol. 2008, Article ID 149286, 12 pages, 2008.

[4] F. Qi, J. Sándor, S. S. Dragomir, and A. Sofo, "Notes on the Schur-convexity of the extended mean values," Taiwanese Journal of Mathematics, vol. 9, no. 3, pp. 411-420, 2005.

[5] H.-N. Shi, S.-H. Wu, and F. Qi, "An alternative note on the Schur-convexity of the extended mean values," Mathematical Inequalities E Applications, vol. 9, no. 2, pp. 219-224, 2006.

[6] D.-M. Li and H.-N. Shi, "Schur convexity and Schur-geometrically concavity of generalized exponent mean," Journal of Mathematical Inequalities, vol. 3, no. 2, pp. 217-225, 2009.

[7] Y. Chu and X. Zhang, "Necessary and sufficient conditions such that extended mean values are Schurconvex or Schur-concave," Journal of Mathematics of Kyoto University, vol. 48, no. 1, pp. 229-238, 2008.

[8] Z.-H. Wang and X.-M. Zhang, "Necessary and sufficient conditions for Schur convexity and Schurgeometrically convexity of Gini means," Communications of Inequalities Researching, vol. 14, no. 2, pp. 193-197, 2007 (Chinese).

[9] Y. Chu and W. Xia, "Solution of an open problem for Schur convexity or concavity of the Gini mean values," Science in China. Series A, vol. 52, no. 10, pp. 2099-2106, 2009.

[10] F. Qi, "A note on Schur-convexity of extended mean values," The Rocky Mountain Journal of Mathematics, vol. 35, no. 5, pp. 1787-1793, 2005.

[11] J. Sándor, "The Schur-convexity of Stolarsky and Gini means," Banach Journal of Mathematical Analysis, vol. 1, no. 2, pp. 212-215, 2007.

[12] Zh.-H. Yang, "Necessary and sufficient conditions for Schur convexity of Stolarsky and Gini means," submitted.

[13] X.-M. Zhang, Geometrically Convex Functions, An'hui University Press, Hefei, China, 2004.

[14] Z.-H. Wang, "The necessary and sufficient condition for S-convexity and S-geometrically convexity of Gini mean," J. Beijing Ins. Edu, vol. 2, no. 5, pp. 1-3, 2007.

[15] Y. Chu, X. Zhang, and G. Wang, "The Schur geometrical convexity of the extended mean values," Journal of Convex Analysis, vol. 15, no. 4, pp. 707-718, 2008.

[16] H.-N. Shi, Y.-M. Jiang, and W.-D. Jiang, "Schur-convexity and Schur-geometrically concavity of Gini means," Computers $\mathcal{E}$ Mathematics with Applications, vol. 57, no. 2, pp. 266-274, 2009.

[17] Ch. Gu and H. N. Shi, "The Schur-convexity and the Schur-geometric concavity of Lehme means," Mathematics in Practice and Theory, vol. 39, no. 12, pp. 183-188, 2009.

[18] A. W. Marshall and I. Olkin, Inequalities: Theory of Majorization and Its Applications, vol. 143 of Mathematics in Science and Engineering, Academic Press, New York, NY, USA, 1979.

[19] B.-Y. Wang, Foundations of Majorization Inequalities, Beijing Normal University Press, Beijing, China, 1990.

[20] C. P. Niculescu, "Convexity according to the geometric mean," Mathematical Inequalities \& Applications, vol. 3, no. 2, pp. 155-167, 2000.

[21] Zh.-H. Yang, "On the homogeneous functions with two parameters and its monotonicity," Journal of Inequalities in Pure and Applied Mathematics, vol. 6, no. 4, article 101, 2005. 
[22] Zh.-H. Yang, "On the log-convexity of two-parameter homogeneous functions," Mathematical Inequalities E Applications, vol. 10, no. 3, pp. 499-516, 2007.

[23] Zh.-H. Yang, "Some monotonictiy results for the ratio of two-parameter symmetric homogeneous functions," The International Journal of Mathematics and Mathematical Sciences, vol. 2009, Article ID 591382, 12 pages, 2009. 


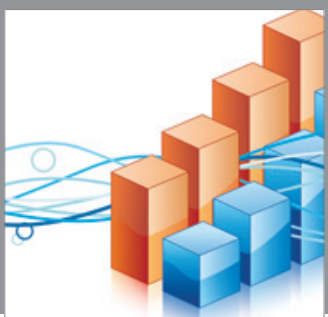

Advances in

Operations Research

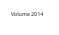

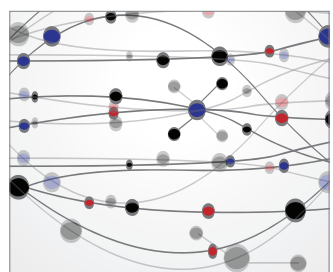

\section{The Scientific} World Journal
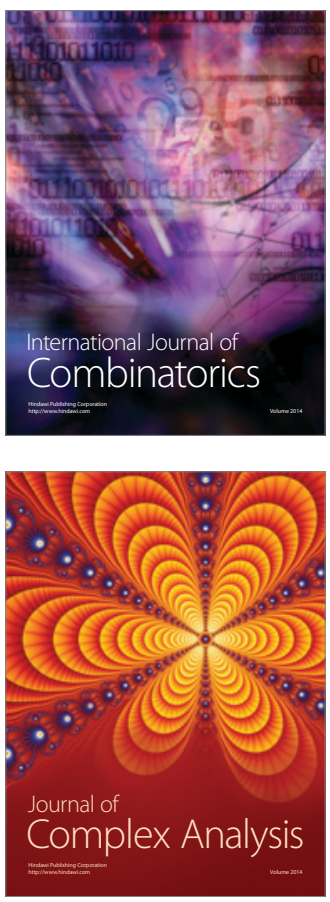

International Journal of

Mathematics and

Mathematical

Sciences
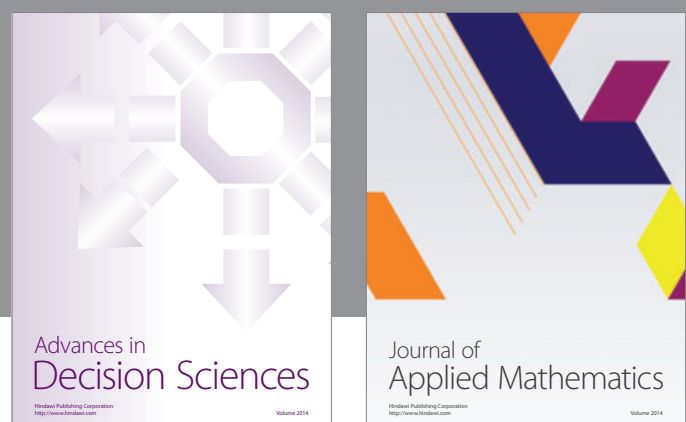

Journal of

Applied Mathematics
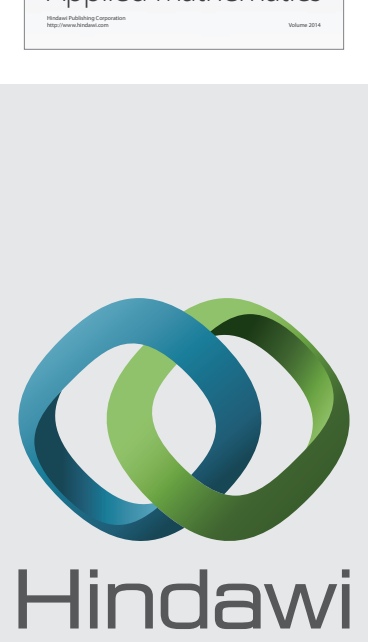

Submit your manuscripts at http://www.hindawi.com
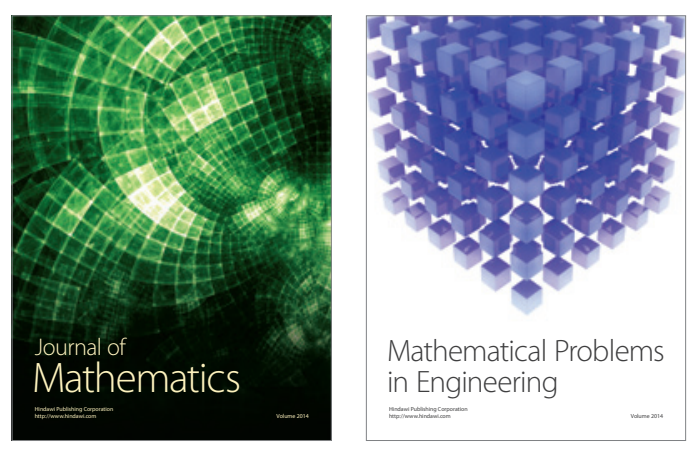

Mathematical Problems in Engineering
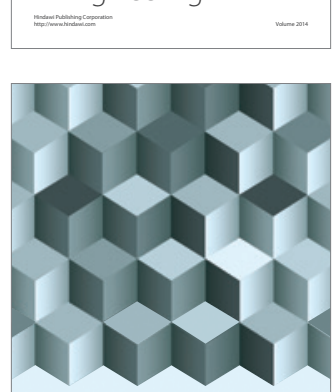

Journal of

Function Spaces
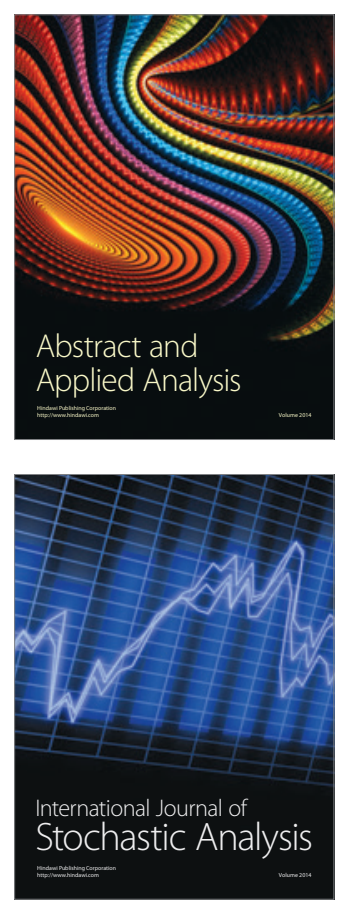

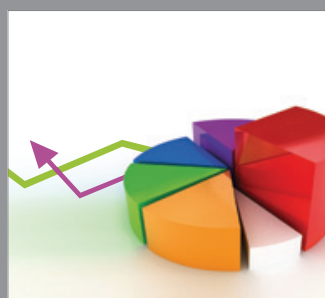

ournal of

Probability and Statistics

Promensencen
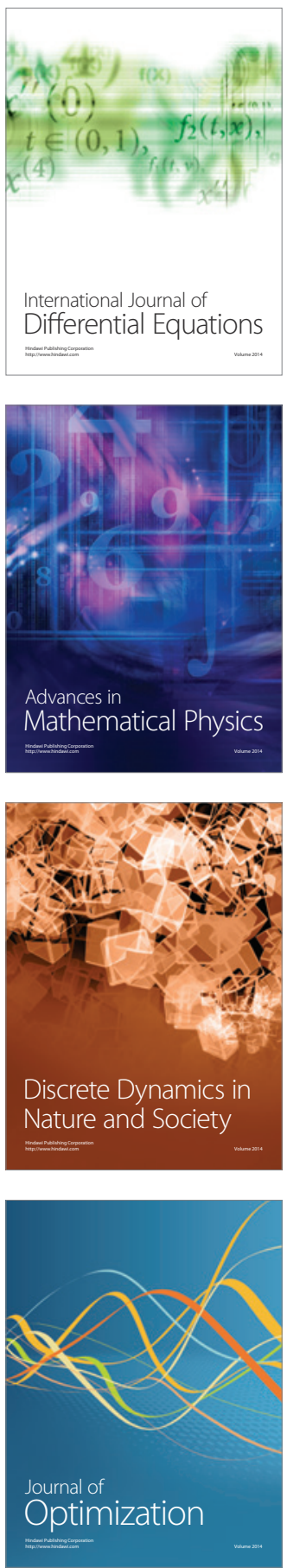